\title{
Pharyngeal-Cervical-Brachial Variant of Guillain-Barre Syndrome
}

\author{
Justin N. Hall a, c, Maryam N. Sheikh ${ }^{\mathrm{a}}$, Steven L. Shumak ${ }^{\mathrm{a}, \mathrm{b}}$
}

\begin{abstract}
Guillain-Barre syndrome (GBS) is the most common cause of acute neuromuscular paralysis in the Western world. We describe the only known case presentation of pharyngeal-cervical-brachial variant following a subacute myocardial infarction and Vibrio cholerae vaccination. A 55-year-old Filipino man presented to our emergency department with a one-day history of progressive oropharyngeal and cervicobrachial weakness and areflexia in the upper limbs. His review of systems was unremarkable except for receiving "Dukoral" traveller's diarrhea vaccine 3 weeks prior. His physical exam was compatible with GBS. EMG and nerve conduction studies showed low motor amplitudes, prolonged distal latency and absent F-waves in the arms. Lumbar puncture showed a protein level of $579 \mathrm{mg} / \mathrm{L}$. Nuclear perfusion scan was consistent with a subacute myocardial infarction. He received a 2-day course of intravenous immunoglobulin. He showed significant improvement in his weakness over 3 days. Recent vaccination and myocardial infarction may be important triggers for GBS onset.
\end{abstract}

Keywords: Guillain-Barre syndrome; Vaccination; Myocardial infarction

\section{Introduction}

Guillain-Barre syndrome (GBS) is the most common cause of acute neuromuscular paralysis in the Western world [1]. The pharyngeal-cervical-brachial (PCB) variant of GBS is characterized by facial palsy, dysarthria, upper extremity muscle weakness and areflexia in the upper limbs [2]. Nerve

\footnotetext{
Manuscript accepted for publication April 14, 2014

${ }^{a}$ Faculty of Medicine, University of Toronto, Toronto, Ontario, Canada ${ }^{\mathrm{b}}$ Division of General Internal Medicine, Department of Medicine, Sunnybrook Health Sciences Centre, Toronto, Ontario, Canada ${ }^{\mathrm{c}}$ Corresponding author: Justin N. Hall, Faculty of Medicine, University of Toronto, 1 King's College Circle, Toronto, Ontario, Canada.

Email: justin.hall@mail.utoronto.ca
}

doi: http://dx.doi.org/10.14740/jnr270w conduction studies suggest that the PCB variant displays axonal rather than demyelinating neuropathy [2]. Infection, inflammation and surgery are commonly reported triggers of GBS and its variants [1]. Individual case reports have reported a potential link between GBS and tetanus, hepatitis and rabies vaccinations $[1,3]$. As the PCB variant is quite rare, it is often misdiagnosed as brainstem stroke, myasthenia gravis, or botulism [2]. We describe the only known case presentation of PCB variant of GBS following a subacute myocardial infarction and Vibrio cholerae vaccination.

\section{Case Report}

A 55-year-old Filipino man presented to our emergency department with a one-day history of progressive quadriparesis, facial weakness and dysphagia. The patient had awakened that day with limited ability to move his arms or hands, weakness in his lower extremities, and dysphagia. He had no previous such symptoms. He had been in the Philippines at the time of symptom onset and flew back to Canada on the day his weakness started. He denied any infectious, constitutional, gastrointestinal, or urinary symptoms. He did endorse some new reflux symptoms 2 days prior to the onset of the muscle weakness. There was no chest pain, dyspnea, or diaphoresis. Review of systems was unremarkable except for receiving "Dukoral" (oral, heat and formalin inactivated Vibrio cholerae traveller's diarrhea and cholera vaccine) 3 weeks prior. The patient was on no CNS drug or biologic therapy. His past medical history was non-contributory.

On physical examination, his heart rate was $112 \mathrm{bpm}$ and his blood pressure was 165/108 despite having no history of hypertension. Normal S1 and S2 heart sounds were heard with no S3, S4, or murmurs appreciated. JVP was 3 $\mathrm{cm}$ ASA. He had normal muscle bulk and tone but decreased cervical flexion and proximal muscle power bilaterally (1 to $2 / 5$ ) in upper extremities (proximal weaker than distal) and lower extremities $(3+$ to $4 / 5)$. He was unable to shrug his shoulders. Reflexes were absent in the upper limbs and preserved in the lower limbs. The patient had mild dysarthria, dysphonia (nasal quality), impaired palatal elevation, with associated facial muscle and tongue muscle weakness 
reflecting weakness of cranial nerves VII, IX, X and XII. His sensory and cerebellar exams were normal.

Brain and spinal cord MRI and CTA chest revealed no abnormalities. Laboratory investigations including $\mathrm{CBC}$, routine chemistry, renal and liver profile, TSH, ESR, CRP, and vitamin B12 were within normal limits. Troponin was elevated and ECG showed ST segment elevation in V1-V3. HSV, syphilis, West Nile virus, HIV, monospot, rheumatoid factor, ANA and Lyme disease serology were negative. EMG and nerve conduction studies showed low motor amplitudes, prolonged distal latency and absent F-waves in the arms. Especially in light of his clinical presentation, these results were considered to be compatible with GBS. A lumbar puncture showed a protein level of $579 \mathrm{mg} / \mathrm{L}$ (normal < $450 \mathrm{mg} / \mathrm{L}$ ) with normal cellularity. A presumptive diagnosis of PCB variant of GBS was made, and the patient received a 2-day course of intravenous immunoglobulin (IVIg). $\mathrm{He}$ showed significant improvement in his weakness over 3 days. Nuclear myocardial perfusion scan was consistent with a subacute anterior myocardial infarction (MI). He was discharged home with outpatient rehabilitation for speech, swallowing, and residual muscle weakness as well as cardiology follow-up. Four weeks following discharge home, our patient received coronary angioplasty of his left anterior descending artery. He continued to have minimal residual facial muscle and tongue muscle weakness but was otherwise back to his baseline. He did not receive any further medications or course of IVIg.

\section{Discussion}

GBS is the most common cause of acute neuromuscular paralysis affecting approximately $0.4-2.4 / 100,000$ people annually [1]. It is characterized by the acute onset of generalized and symmetrical muscle weakness and areflexia in an ascending pattern. There are several less common variants of GBS that have unusual patterns of muscle involvement. The PCB variant of GBS is rare $(0.07-0.25 / 100,000)$ [4], and it is characterized by oropharynx, neck and proximal upper limb muscle weakness $[5,6]$. Antecedent infection with CMV or Campylobacter jejuni is often found [1] with anti-GM2 antibodies being a hallmark of this variant [1, 7]. Further diagnostic criteria for PCB variant include the presence of diaphragm weakness, relative sparing of lower limb strength and tendon reflexes, minimal or no sensory deficit, raised CSF protein, and generally axonal neuropathy on nerve conduction studies $[6,8]$. In most cases, patients experience a complete recovery $[2,6]$.

Our patient's case illustrates many of the classic characteristics of PCB variant including areflexia, facial palsy, dysarthria and upper extremity proximal muscle weakness. While influenza vaccination has been associated with GBS [9] and individual case reports have reported a potential link between GBS and tetanus, hepatitis and rabies vaccinations $[1,3]$, the authors are unaware of any published cases describing an association between PCB variant and vaccinations, including those against Vibrio cholerae which our patient had received 3 weeks before his symptoms developed. The reflux symptoms, hypertension, tachycardia and lack of chest pain associated with a recent myocardial infarction are consistent with dysautonomia as a complication of our patient's GBS. While infection, inflammation and surgery are commonly reported triggers of GBS [1], only three cases have been reported where an MI was the trigger for onset of GBS without treatment with streptokinase [10]. No known cases of PCB variant following an MI have been reported. We wish to raise awareness of this rare condition so that physicians consider PCB variant of GBS in patients with symptoms of cranial nerve and upper extremity weakness as diagnosis and early intervention greatly benefit the patient's eventual outcome.

\section{Funding Source}

None.

\section{Conflict of Interest}

The authors do not have any conflicts of interest to declare.

\section{References}

1. Hughes RA, Cornblath DR. Guillain-Barre syndrome. Lancet. 2005;366(9497):1653-1666.

2. Wakerley BR, Yuki N. Pharyngeal-cervical-brachial variant of Guillain-Barre syndrome. J Neurol Neurosurg Psychiatry. 2014;85(3):339-344.

3. van Doorn PA, Ruts L, Jacobs BC. Clinical features, pathogenesis, and treatment of Guillain-Barre syndrome. Lancet Neurol. 2008;7(10):939-950.

4. Guillain-Barre syndrome variants in Emilia-Romagna, Italy, 1992-3: incidence, clinical features, and prognosis. Emilia-Romagna Study Group on Clinical and Epidemiological Problems in Neurology. J Neurol Neurosurg Psychiatry. 1998;65(2):218-224.

5. Ropper AH. Unusual clinical variants and signs in Guillain-Barre syndrome. Arch Neurol. 1986;43(11):11501152.

6. Nagashima T, Koga M, Odaka M, Hirata K, Yuki N. Continuous spectrum of pharyngeal-cervical-brachial variant of Guillain-Barre syndrome. Arch Neurol. 2007;64(10):1519-1523.

7. Yuki N, Tagawa Y. Acute cytomegalovirus infection and IgM anti-GM2 antibody. J Neurol Sci. 1998;154(1):14- 
17.

8. Ropper AH, Wijdicks EFM, Truax BT. Guillain-Barre Syndrome.Philadelphia: FA Davis Company Publishers; 1991.

9. Juurlink DN, Stukel TA, Kwong J, Kopp A, McGeer A,
Upshur RE, Manuel DG, et al. Guillain-Barre syndrome after influenza vaccination in adults: a population-based study. Arch Intern Med. 2006;166(20):2217-2221.

10. Ng E, Stafford PJ. Guillain-Barre syndrome after myocardial infarction. Int J Cardiol. 2003;90(1):129-130. 